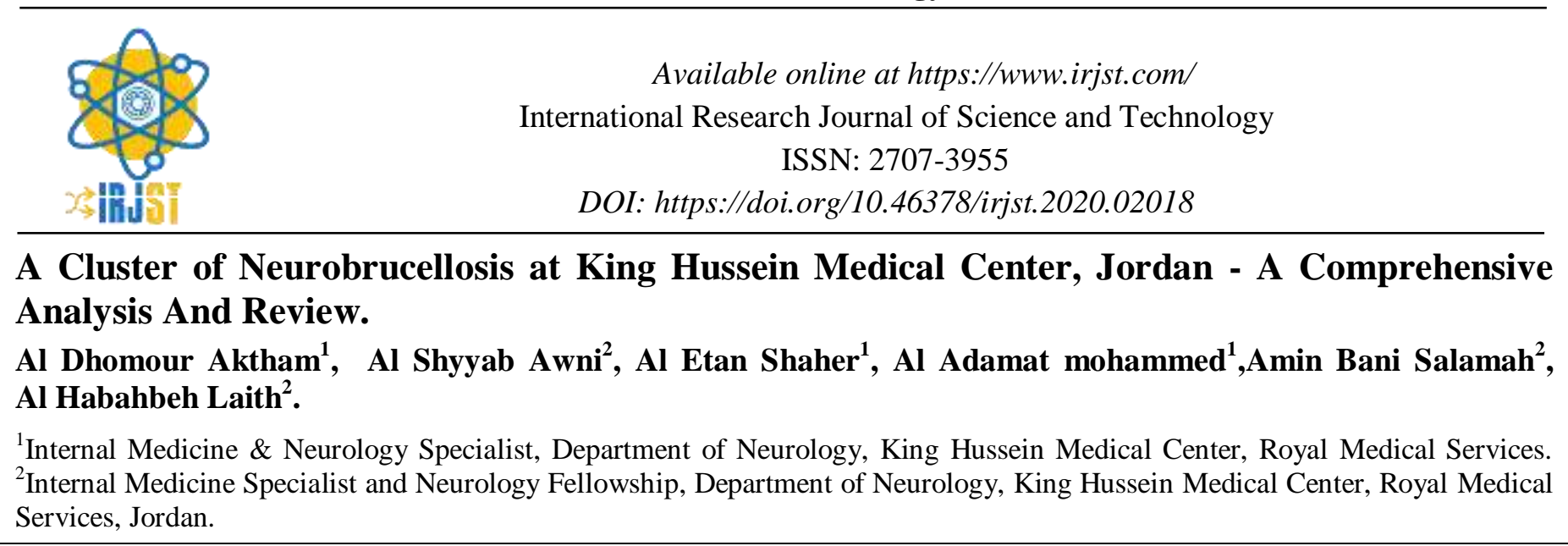

\title{
A Cluster of Neurobrucellosis at King Hussein Medical Center, Jordan - A Comprehensive
} Analysis And Review.

\author{
Al Dhomour Aktham1, Al Shyyab Awni ${ }^{2}$, Al Etan Shaher ${ }^{1}$, Al Adamat mohammed ${ }^{1}$,Amin Bani Salamah ${ }^{2}$, \\ Al Habahbeh Laith ${ }^{2}$. \\ ${ }^{2}$ Internal Medicine Specialist and
}

\begin{tabular}{lll}
\multicolumn{2}{l}{ Paper Status } & \\
\hline Received & Nov 2020 \\
Accepted & Dec 2020 \\
Published & $:$ & Dec 2020
\end{tabular}

Key Words

Brucellosis,

Neurobrucellosis,

Pseudotumor Cerebri

\begin{abstract}
In this retrospective review, we describe the neurological clinical manifestations in five cases diagnosed with neurobrucellosis over two years between 2018 and 2020, and the application of different proposed criteria for establishing the diagnosis and treatment of neurobrucellosis. All cases were confirmed to have brucellosis with laboratory tests, and all were living in Jordan, which is considered part of a highly endemic area within the Middle East. The neurobrucellosis proposed criteria was applied, which requires signs and symptoms consistent with neurobrucellosis, presence of anti-Brucella antibodies in cerebrospinal fluid and serum with or without isolation of Brucella species in CSF and serum, cerebrospinal fluid analysis shows: lymphocytosis, high protein level, and low glucose level and radiological findings on MRI or CT. In all five cases, we have confirmed direct contact with animals or animal products either as a consumer or as a farmworker. Their signs and symptoms were consistent with brucellosis. In all cases, haematological and CSF results, in addition to imaging findings using magnetic resonance and computed tomography, were highly coherent with neurobrucellosis. Neurobrucellosis can present with different clinical manifestations, either as a sole site of infection like acute or subacute meningitis or myelitis, or in context of a multi variable systemic disease. In patients with unusual neurological clinical presentations, and those with persistent chronic symptoms like headache, malaise, or depression, neurobrucellosis should be highly suspected, especially in endemic areas. Such patients must have a prolonged course of antibiotics between 6 to 18 months with close monitoring of their serum and CSF examination.
\end{abstract}

Copyright () 2020: Al Dhomour Aktham, Al Shyyab Awni, Al Etan Shaher, Al Adamat mohammed, Amin Bani Salamah, Al Habahbeh Laith. This is an open-access distribution, and reproduction in any medium provided Access article distributed under the Creative Commons Attribution License the original work is properly cited License, which permits unrestricted use.

Citation: Al Dhomour Aktham, Al Shyyab Awni, Al Etan Shaher, Al Adamat mohammed, Amin Bani Salamah, Al Habahbeh Laith. "A Cluster of Neurobrucellosis at King Hussein Medical Center, Jordan - A Comprehensive Analysis And Review". International Research Journal of Science and Technology, 2 (1), 349-358, 2020.

\section{Introduction}

Brucellosis is the most common bacterial zoonosis around the world, with more than half a million new cases registered annually. In Jordan, brucellosis is still endemic, with an annual incidence of more than 300 cases per million [1,2], especially in rural areas that houses high numbers of refugees with high animal population density.

\footnotetext{
* Corresponding Author: Al Dhomour Aktham

Internal Medicine \& Neurology Specialist, Department of Neurology, King Hussein Medical Center, Royal Medical Services, Jordan. Email: akthamful12@gmail.com
}

According to the ministry of health and the ministry of agriculture, there has been an increase in the numbers of new cases diagnosed with Brucella in recent years, as a result of the low vaccination coverage for animals and the inadequate information about the modes of the disease transmission, with seasonal incidence being higher in spring and summer months.

Brucellosis is caused by the bacteria genus Brucella, with predominance of Brucella melitensis and abortus in Jordan, according to Jordan Ministry of Health $(\mathrm{MoH})$ and Jordan Ministry of Agriculture 
(MoA) [3, 4, 5]. Brucella can be transmitted via contact with infected animals or animal products contaminated with Brucella bacteria, for example, ingestion of unpasteurized milk or undercooked meat. Other modes of transmission are via inhalation and percutaneous through minor wounds or through mucous membranes. Brucellosis is not transmitted between humans via close contact, but few anecdotal cases were reported[6]. Neurobrucellosis is a rare complication, and it can develop at any stage of the disease [7]. It may manifest with protean signs and symptoms, like fever, headache, vomiting, photophobia with less frequent neck rigidity, focal neurological deficit, and sensory symptoms which are estimated to occur in up to $25 \%$ [27]. It may run from an acute to chronic course, and have variable presentations like: acute, subacute and chronic meningitis, subarachnoid haemorrhage, encephalitis, myelitis [27], Meningoencephalitis, Stroke and peripheral neuropathy. Other rare manifestations include: pseudotumor cerebri, intracranial granuloma, sagittal sinus thrombosis, arachnoiditis, and intracranial vasculitis $[8,9,12,13,14,17]$.

Neurobrucellosis is a perplexing diagnosis[ with an estimated incidence of $1.7 \%-10 \% 10,30$ ], although its incidence has reached up to $30 \%$ depending on some cohort studies[36]. Its diagnosis is fundamentally based on the clinical signs and symptoms which are not explained by any other neurological disease, plus an alteration of the results of cerebrospinal fluid and hematological sampling, along with imaging and radiological findings. Neurobrucellosis is a diagnostic riddle due to limited assent criteria [11]. On the one hand, it may be related to the presence of nonspecific symptoms, and on the other hand, it is due to the lack of diagnostic facilities, which lead to a delay in reaching a diagnosis for up to 6 months or even longer in some cases $[15,16]$. Neurobrucellosis may carry a high risk of morbidity and mortality if not treated early. A favorable outcome can be achieved with a suitable approach and ploy-antibiotic therapy, with the duration of treatment depending mainly on improvement of the neurological manifestations and clearance of the cerebrospinal fluid.

\section{Materials and Methods}

We conducted this retrospective review in the neurology department at King Hussein medical center, a tertiary neurology referral center. We have reviewed the medical records for all of the five cases. Clinical details, laboratory data, and imaging reports of the presented cases were evaluated and analyzed. First presentation, duration and course of illness, epidemiological and occupational history, consuming unpasteurized milk and milk products, and if being exposed to infected animals were all gathered and documented. Three out of five cases presented with bilateral papilledema, one case presented with myelitis, and another patient had an ischemic strokein Table.1. Case 1, was a 49 years old female, medically free, housewife as mentioned in table.2. Her initial symptoms were malaise, fatigue, and arthritis following a chronic course. At the beginning of 2018 her clinical examination was unremarkable, and all of her laboratory investigations were within normal limits. She was treated at a primary healthcare clinic with simple analgesics. However, few months later her symptoms worsened and she started to have a headache, depression, low-grade fever, and weight loss. At that time, ESR was $100 \mathrm{~mm} / 1^{\text {st }}$ hour and her brain computed tomography, abdominal ultrasound and the thyroid function test were reported as normal.

In August 2018, patient developed blurred vision and decreased visual acuity and thus she was sent to ophthalmology clinic for consultation, where she was diagnosed with bilateral optic disc swelling (grade II papilledema) and was started on oral acetazolamide. At this point, she was referred to our clinic with a request to do a CSF manometer to measure CSF pressure, after brain MRI plus MRV were done and revealed no findings. CSF manometer showed a high CSF opening pressure of $>49 \mathrm{cmH}_{2} \mathrm{O}$, and CSF analysis came back with WBC $100 / \mathrm{mm}^{3}$ (lymphocytes > 95\%), high protein $289 \mathrm{mg} / \mathrm{dL}$ and glucose 37 $\mathrm{mg} / \mathrm{dL}$. CSF culture and PCR for tuberculosis were negative. Brucella test, the serum (tube) agglutination test (SAT) was positive with Brucella IgG antibody titer $1 / 320$.

The patient was started on triple antibiotic therapy for chronic Brucella meningitis (Ceftriaxone 2g IV daily for three weeks replaced then by trimethoprimsulfamethoxazole $400 / 80 \mathrm{mg}$ orally twice daily, rifampicin $600 \mathrm{mg} /$ daily, and doxycycline $100 \mathrm{mg}$ bid/ daily) for four months. After four months (in January 2019), Brucella titer was $1 / 160$, ESR $85 \mathrm{ml} /$ hour, High $>20 \mathrm{mg} / \mathrm{dl}$ and CSF analysis was repeated and showed dramatic improvement: WBC $50 / \mathrm{mm}^{3}$ (lymphocytes $95 \%$ ), protein $100 \mathrm{mg} / \mathrm{dL}$ and glucose $56 \mathrm{mg} / \mathrm{dL}$, and ICP $21 \mathrm{cmH}_{2} \mathrm{O}$. In September 2019 CSF: WBC 0$5 / \mathrm{mm}^{3}$, glucose $85 \mathrm{mg} / \mathrm{dL}$, protein $90 \mathrm{mg} / \mathrm{dL}$, Brucella titer 1/160, CRP high > 20mg/dl and ESR $46 \mathrm{~mm} / 1^{\text {st }}$ hour. All patient symptoms were resolved, and after a regular follow-up at the neuro-ophthalmology clinic, acetazolamide stopped. The decision was taken to continue treatment with the same regime until her CSF analysis is back to normal. In March 2020, the patient had a lumbar puncture done besides other laboratory investigations, and her results ultimately returned back to normal. CSF analysis (WBC $0-5 \mathrm{~mm}^{3}$, glucose $65 \mathrm{mg} / \mathrm{dl}$, Protein $45 \mathrm{mg} / \mathrm{dL}$ ), Brucella titer $1 / 80$, ESR $25 \mathrm{~mm} / 1^{\text {st }} \mathrm{hr}$ and CRP was normal. Treatment was discontinued with total duration of therapy, in this case, being around 18+ months. 
Table 1. Summary of signs and symptoms, laboratory findings, cerebrospinal fluid analysis and radiological findings.

\begin{tabular}{|c|c|c|c|c|c|c|c|}
\hline Case & Age & Gender & Occupation & $\begin{array}{l}\text { Symptoms, signs } \\
\text { \&duration } \\
\text { Diagnosis }\end{array}$ & $\begin{array}{l}\text { Brucella test } \\
\text { /titer }\end{array}$ & $\begin{array}{l}\text { CSF } \\
\text { analysis }\end{array}$ & $\begin{array}{l}\text { Images } \\
\text { MRI }\end{array}$ \\
\hline Case 1 & 49 & Female & Housewife & $\begin{array}{l}\text { Low grade fever, } \\
\text { headache } \\
\text { Blurred } \\
\text { vision, Malaise, } \\
\text { fatigue, arthralgia, } \\
\text { weight loss.> } 6 \\
\text { weeks } \\
\text { Bilateral optic disc } \\
\text { swelling. } \\
\text { Pseudotumor cerebri } \\
\text { \& chronic meningitis }\end{array}$ & $\begin{array}{l}\text { Positive } \\
\text { serum tube } \\
\text { agglutination } \\
\text { test with titer } \\
>1 / 320\end{array}$ & $\begin{array}{l}\text { WBC } \\
100 \mathrm{~mm}^{3}, \\
95 \% \\
\text { lymphocytes. } \\
\text { Protein } 289 \\
\text { mg/dl } \\
\text { Glucose } 37 \\
\text { mg/dl } \\
\text { Culture } \\
\text { negative } \\
\text { T.B PCR } \\
\text { negative } \\
\text { ICP > 49 } \\
\text { mmHg }\end{array}$ & $\begin{array}{l}\text { Normal } \\
\text { brain MRI / } \\
\text { MRV\& } \\
\text { brain CT } \\
\text { scan }\end{array}$ \\
\hline Case 2 & 32 & Male & $\begin{array}{l}\text { Office } \\
\text { worker }\end{array}$ & $\begin{array}{l}\text { Headache, blurred } \\
\text { vision, vomiting.> } 4 \\
\text { weeks } \\
\text { Mild nuchal rigidity } \\
\text { Bilateral optic disc } \\
\text { swelling. } \\
\text { Pseudotumor cerebri } \\
\& \\
\text { subacute meningitis }\end{array}$ & $\begin{array}{l}\text { Positive } \\
\text { serum tube } \\
\text { agglutination } \\
\text { test with titer } \\
>1 / 320\end{array}$ & $\begin{array}{l}\text { WBC } \\
390 \mathrm{~mm}^{3}, \\
95 \% \\
\text { lymphocytes. } \\
\text { Protein } 156 \\
\text { mg/dl } \\
\text { Glucose } 40 \\
\text { mg/dl } \\
\text { Culture } \\
\text { negative } \\
\text { T.B PCR } \\
\text { negative } \\
\text { ICP > 32 } \\
\text { cmH2o }\end{array}$ & $\begin{array}{l}\text { Brain } \\
\text { MRI: mild } \\
\text { dilated } \\
\text { ventricular } \\
\text { system, } \\
\text { tortuous } \\
\text { optic disc }\end{array}$ \\
\hline Case 3 & 23 & Female & $\begin{array}{l}\text { School } \\
\text { teacher }\end{array}$ & $\begin{array}{l}\text { Headache, blurred } \\
\text { vision low grade } \\
\text { fever, joint pain } \\
>4 \text { weeks } \\
\text { Mild nuchal rigidity } \\
\text { Bilateral optic disc } \\
\text { swelling. } \\
\text { Pseudotumor cerebri } \\
\& \\
\text { subacute meningitis }\end{array}$ & $\begin{array}{l}\text { Positive } \\
\text { Rose Bengal } \\
\text { test \& serum } \\
\text { tube } \\
\text { agglutination } \\
\text { with titer > } \\
1 / 320\end{array}$ & $\begin{array}{l}\text { WBC } \\
60 \mathrm{~mm}^{3}, 90 \% \\
\text { lymphocytes. } \\
\text { Protein } 115 \\
\mathrm{mg} / \mathrm{dl} \\
\text { Glucose } 30 \\
\mathrm{mg} / \mathrm{dl} \\
\text { Culture } \\
\text { negative } \\
\text { T.B PCR } \\
\text { negative } \\
\text { ICP >50 } \\
\mathrm{cmH2o}\end{array}$ & $\begin{array}{l}\text { Brain } \\
\text { MRI: mild } \\
\text { dilated } \\
\text { ventricular } \\
\text { system, } \\
\text { tortuous } \\
\text { optic disc }\end{array}$ \\
\hline Case 4 & 39 & Male & Soldier & $\begin{array}{l}\text { Headache, low grade } \\
\text { fever, Left upper and } \\
\text { lower limbs weakness } \\
>4 \text { weeks } \\
\text { Stroke. }\end{array}$ & $\begin{array}{l}\text { Positive } \\
\text { serum tube } \\
\text { agglutination } \\
\text { test with titer } \\
>1 / 640\end{array}$ & $\begin{array}{l}\text { WBC } \\
85 \mathrm{~mm}^{3}, \\
90 \% \\
\text { lymphocytes. } \\
\text { Protein } 111 \\
\text { mg/dl } \\
\text { Glucose } 37 \\
\text { mg/dl } \\
\text { Culture } \\
\text { negative } \\
\text { T.B PCR } \\
\text { negative } \\
\text { ICP < 15 } \\
\text { cmH2o }\end{array}$ & $\begin{array}{l}\text { Brain CT } \\
\text { scan \& MRI: } \\
\text { ischemic } \\
\text { infarction of } \\
\text { the posterior } \\
\text { aspect of } \\
\text { right } \\
\text { temporal } \\
\text { lobe }\end{array}$ \\
\hline
\end{tabular}




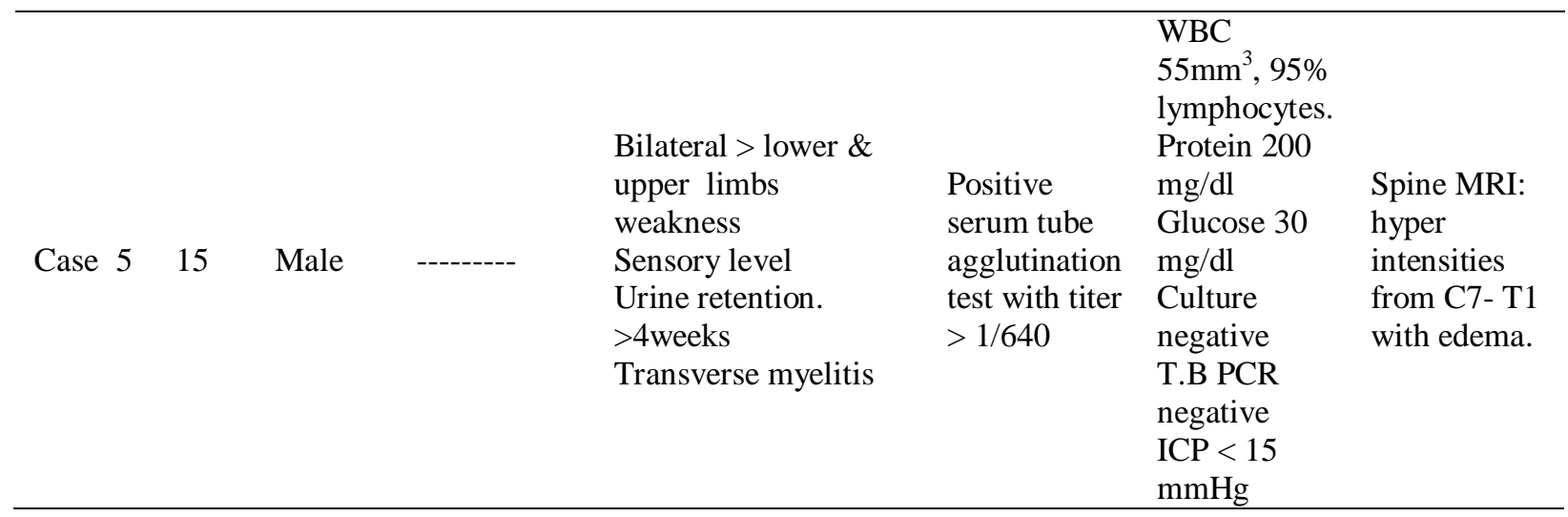

Table 2. Summary of laboratory results throughout the treatment course and the follow-up period (August 2018- March 2020). (Case 1)

\begin{tabular}{|c|c|c|c|c|}
\hline Investigation/ Date & August 2018 & January 2019 & September 2019 & March 2020 \\
\hline \multirow{5}{*}{ CSF analysis } & WBC $100 \mathrm{~mm}^{3}$ & WBC $50 \mathrm{~mm}^{3}$ & & WBC $0-5 \mathrm{~mm}^{3}$ \\
\hline & (lymphocytes 95\%) & (lymphocytes & WBC $0-5 \mathrm{~mm}^{3}$ & Protein \\
\hline & Protein $289 \mathrm{mg} / \mathrm{dL}$ & $95 \%)$ & Protein $90 \mathrm{mg} / \mathrm{dl}$ & $45 \mathrm{mg} / \mathrm{dL}$ \\
\hline & Glucose $37 \mathrm{mg} / \mathrm{dL}$ & Protein $100 \mathrm{mg} / \mathrm{dL}$ & Glucose 85 & \\
\hline & $\mathrm{ICP}>49 \mathrm{cmH}_{2} \mathrm{O}$ & $\begin{array}{l}\text { Glucose } 56 \mathrm{mg} / \mathrm{dL} \\
\text { ICP } 21 \mathrm{cmH}_{2} \mathrm{O}\end{array}$ & $\mathrm{mg} / \mathrm{dl}$ & $\begin{array}{l}\text { Glucose } \\
65 \mathrm{mg} / \mathrm{dL}\end{array}$ \\
\hline Brucella test $\&$ titer & Positive $-1 / 320$ & Positive - 1/160 & Positive - 1/160 & Positive - 1/80 \\
\hline CSF culture & No growth & No growth & No growth & No growth \\
\hline TB PCR & Negative & ------ & --------- & ------ \\
\hline $\mathrm{CBC}$ & Normal & & Normal & Normal \\
\hline Chemistry & Normal & ------- & Normal & Normal \\
\hline ESR & $100 \mathrm{~mm} / 1^{\text {st }}$ hour & $85 \mathrm{~mm} / 1^{\text {st }}$ hour & $46 \mathrm{~mm} / 1^{\mathrm{st}}$ hour & $25 \mathrm{~mm} / 1^{\text {st }}$ hour \\
\hline CRP & High> $39.0 \mathrm{mg} / \mathrm{dl}$ & High $>20 \mathrm{mg} / \mathrm{dl}$ & ----------- & Normal \\
\hline
\end{tabular}

CSF: Cerebrospinal Fluid, T.B PCR: Polymerase Chain Reaction for Mycobacterium Tuberculosis, CBC: Complete blood count, CRP: C- Reactive Protein, ESR: Erythrocyte Sedimentation Rate.

Table 3. Summary of laboratory results throughout treatment and follow up period (June2019- February 2020) (Case 2)

\begin{tabular}{|c|c|c|c|}
\hline Investigation/ Date & June 2019 & September 2019 & February 2020 \\
\hline \multirow{4}{*}{ CSF analysis } & $\begin{array}{l}\text { WBC 390mm } \\
\text { (lymphocytes 95\%) }\end{array}$ & $\begin{array}{l}\text { WBC 50mm } \\
\text { (lvmphocytes } 90 \% \text { ) }\end{array}$ & WBC $0-5 \mathrm{~mm}^{3}$ \\
\hline & Protein $156 \mathrm{mg} / \mathrm{dL}$ & Protein $97 \mathrm{mg} / \mathrm{dL}$ & Protein $40 \mathrm{mg} / \mathrm{dL}$ \\
\hline & Glucose $40 \mathrm{mg} / \mathrm{dL}$ & Glucose $77 \mathrm{mg} / \mathrm{dL}$ & Glucose $65 \mathrm{mg} / \mathrm{dL}$ \\
\hline & ICP $32 \mathrm{cmH}_{2} \mathrm{O}$ & ICP $17 \mathrm{cmH}_{2} \mathrm{O}$ & \\
\hline Brucella test $\&$ titer & Positive - 1/320 & Positive - 1/160 & Positive - 1/80 \\
\hline CSF culture & No growth & No growth & No growth \\
\hline TB PCR in CSF & Negative & --------- & --------- \\
\hline $\mathrm{CBC}$ & Normal & Normal & --------- \\
\hline Chemistry & Normal & ---------- & --------- \\
\hline ESR & $25 \mathrm{~mm} / 1^{\text {st }}$ hour & ---------- & --------- \\
\hline CRP & Normal & ---------- & --------- \\
\hline
\end{tabular}

CSF: Cerebrospinal Fluid, T.B PCR: Polymerase Chain Reaction for Mycobacterium Tuberculosis, CBC: Complete blood count, CRP: C- Reactive Protein, ESR: Erythrocyte Sedimentation Rate. 
Table 4._Summary of laboratory results throughout treatment and follow-up period (November 2019- June 2020) (Case 3)

\begin{tabular}{|c|c|c|c|}
\hline Investigation/Date & November 2019 & February 2020 & June 2020 \\
\hline \multirow{4}{*}{ CSF analysis } & $\begin{array}{l}\text { WBC 60mm } \\
\text { (lymphocytes 90\%) }\end{array}$ & $\begin{array}{l}\text { WBC } 12 \mathrm{~mm}^{3} \\
\text { (lymphocytes } 90 \% \text { ) }\end{array}$ & WBC $0-5 \mathrm{~mm}^{3}$ \\
\hline & Protein $115 \mathrm{mg} / \mathrm{dL}$ & Protein $68 \mathrm{mg} / \mathrm{dL}$ & Protein $23 \mathrm{mg} / \mathrm{dL}$ \\
\hline & Glucose $30 \mathrm{mg} / \mathrm{dL}$ & Glucose $39 \mathrm{mg} / \mathrm{dL}$ & Glucose $45 \mathrm{mg} / \mathrm{dL}$ \\
\hline & ICP $50 \mathrm{cmH}_{2} \mathrm{O}$ & ICP $16 \mathrm{cmH}_{2} \mathrm{O}$ & \\
\hline Brucella test \& & Positive - 1/320 & Positive - 1/160 & Positive - 1/80 \\
\hline CSF culture & No growth & No growth & No growth \\
\hline TB PCR in CSF & Negative & ---- & ----------- \\
\hline $\mathrm{CBC}$ & Consistent with & & Within normal \\
\hline & thalassemia trait & & limits \\
\hline Chemistry & Normal & ----------- & Normal \\
\hline ESR & $5 \mathrm{~mm} / 1^{\text {st }}$ hour & ----------- & ----------- \\
\hline CRP & Normal & ------------ & ------------ \\
\hline
\end{tabular}

CSF: Cerebrospinal Fluid, T.B PCR: Polymerase Chain Reaction for Mycobacterium Tuberculosis, CBC: Complete blood count, CRP: C- Reactive Protein, ESR: Erythrocyte Sedimentation Rate.

Table 5. Summary of laboratory results throughout treatment and follow-up period (July 2019- July 2020). (Case 4)

\begin{tabular}{|c|c|c|c|}
\hline Investigation/Date & July 2019 & December 2019 & July 2020 \\
\hline CSF analysis & $\begin{array}{l}\text { WBC } 85 \mathrm{~mm}^{3} \\
\text { (80\% lymphocytes, } 20 \% \\
\text { neutrophils) } \\
\text { Protein } 111 \mathrm{mg} / \mathrm{dL} \\
\text { Glucose } 37 \mathrm{mg} / \mathrm{dL}\end{array}$ & $\begin{array}{l}\text { WBC } 25 \mathrm{~mm}^{3} \\
\text { (all lymphocytes) } \\
\text { Protein } 58 \mathrm{mg} / \mathrm{dL} \\
\text { Glucose } 67 \mathrm{mg} / \mathrm{dL}\end{array}$ & $\begin{array}{l}\text { WBC } 0-5 \mathrm{~mm}^{3} \\
\text { Protein } 38 \mathrm{mg} / \mathrm{dL} \\
\text { Glucose } 67 \mathrm{mg} / \mathrm{dL}\end{array}$ \\
\hline Brucella test \& titer & Positive - 1/640 & Positive - 1/160 & Positive - 1/20 \\
\hline CSF culture & No growth & No growth & No growth \\
\hline TB PCR in CSF & Negative & ----------- & ----------- \\
\hline $\mathrm{CBC}$ & Normal & Normal & Normal \\
\hline Chemistry & Normal & Normal & Normal \\
\hline ESR & $35 \mathrm{~mm} / 1^{\text {st }}$ hour & ---------- & ----------- \\
\hline CRP & Normal & ---------- & ------------ \\
\hline
\end{tabular}

CSF: Cerebrospinal Fluid, T.B PCR: Polymerase Chain Reaction for Mycobacterium Tuberculosis, CBC: Complete blood count, CRP: C- Reactive Protein, ESR: Erythrocyte Sedimentation Rate.

Table 6. Summary of laboratory results throughout treatment and follow-up period (December 2019- June 2020). 9Case 5)

\begin{tabular}{|c|c|c|c|c|}
\hline Investigation/ Date & December 2018 & May 2019 & November 2019 & June 2020 \\
\hline CSF analysis & $\begin{array}{l}\text { WBC 50mm } \mathrm{mm}^{3} \\
\text { (95\%Lymphocytes) } \\
\text { Protein } 200 \mathrm{mg} / \mathrm{dL} \\
\text { Glucose } 32 \mathrm{mg} / \mathrm{dL}\end{array}$ & $\begin{array}{l}\text { WBC } 28 \mathrm{~mm}^{3} \\
\text { (all mononuclear) } \\
\text { Protein } 109 \mathrm{mg} / \mathrm{dL} \\
\text { Glucose } 45 \mathrm{mg} / \mathrm{dL}\end{array}$ & $\begin{array}{l}\text { WBC } 0-5 \mathrm{~mm}^{3} \\
\text { Protein } 76 \mathrm{mg} / \mathrm{dL} \\
\text { Glucose } 65 \mathrm{mg} / \mathrm{dL}\end{array}$ & $\begin{array}{l}\text { WBC } 0-5 \mathrm{~mm}^{3} \\
\text { Protein } 45 \mathrm{mg} / \mathrm{dL} \\
\text { Glucose } 59 \mathrm{mg} / \mathrm{dL}\end{array}$ \\
\hline Brucella test \& titer & $\begin{array}{l}\text { Positive } \\
\text { (isolated Brucella } \\
\text { melitensis) } \\
\text { Antibody titer 1/640 }\end{array}$ & Positive - 1/320 & Positive - 1/160 & Positive - 1/160 \\
\hline CSF culture & No growth & No growth & No growth & No growth \\
\hline $\begin{array}{l}\text { TB PCR in CSF } \\
\text { CBC }\end{array}$ & $\begin{array}{l}\text { Negative } \\
\text { Normal }\end{array}$ & Normal & Normal & ------------ \\
\hline Chemistry & Normal & & Normal & Normal \\
\hline ESR & $51 \mathrm{~mm} / 1^{\text {st }}$ hour & ----------- & ------------- & $20 \mathrm{~mm} / 1^{\mathrm{st}} \mathrm{hr}$ \\
\hline CRP & Normal & -------------- & ------------ & Normal \\
\hline
\end{tabular}

CSF: Cerebrospinal Fluid, T.B PCR: Polymerase Chain Reaction for Mycobacterium Tuberculosis, CBC: Complete blood count, CRP: C- Reactive Protein, ESR: Erythrocyte Sedimentation Rate. 

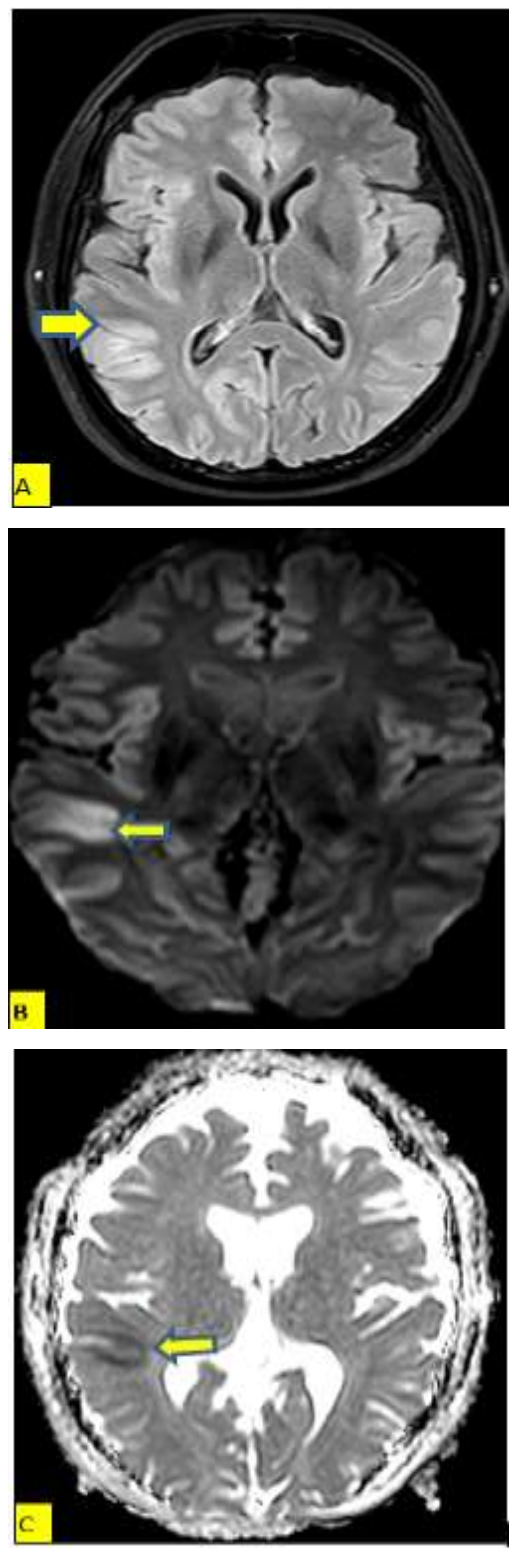

Fig.1.(A) Flair, (B) DWI, (C) ADC. Brain MRI 2019 showed acute ischemic infarction involving the posterior aspect of the right temporal lobe(yellow arrows).

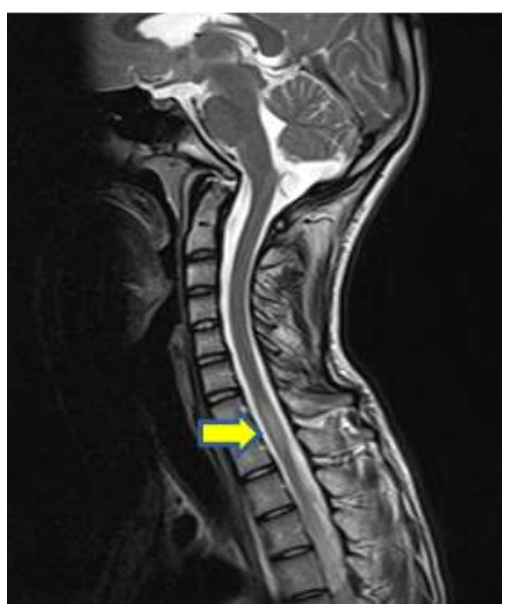

Fig.2. Cervical spine MRI 2018. T2 showed hyperintensities at the spinal cord from C7-T1 with associated cord oedema (yellow arrow).
Case2, a 32 years male patient, he was medically free, an office worker as mentioned in table 3. In June 2019, he was admitted to hospital at neurology ward complaining of a constant diffuse headache, associated with nausea and blurred vision for the last few weeks before admission. Physical examination revealed mild neck rigidity and bilateral optic disc swelling. His brain MRI showed mildly dilated ventricular system and tortuous optic discs, and MRV was reported as normal. His CSF white blood cell count was $390 \mathrm{~mm}^{3}$ (lymphocytes 95\%), Protein $156 \mathrm{mg} / \mathrm{dL}$, glucose 40 $\mathrm{mg} / \mathrm{dL}$. CSF culture and CSF TB PCR were negative. ICP was above $32 \mathrm{cmH}_{2} \mathrm{O}$, ESR $25 \mathrm{~mm} / 1^{\text {st }}$ hour, negative CRP and positive Brucella test with Brucella titer $1 / 320$. We started the patient on the same regimen of triple antimicrobial treatment as in case 1, and all symptoms improved over weeks.

In September 2019, the patient was followed up, CSF analysis was repeated where WBC count dropped to 50 (lymphocytes $90 \%$ ), protein $97 \mathrm{mg} / \mathrm{dL}$, glucose 77 $\mathrm{mg} / \mathrm{dL}$ and ICP dropped to $17 \mathrm{cmH}_{2} \mathrm{O}$. Brucella test remained positive with antibody titer $1 / 160$. A decision to continue treatment was made until complete recovery of the CSF parameters is achieved.

In February 2020, a new CSF analysis was done showing complete resolution of the inflammatory process where all parameters returned back to normal with normal WBC, protein $40 \mathrm{mg} / \mathrm{dL}$ and glucose 65 $\mathrm{mg} / \mathrm{dL}$. Brucella antibody titer dropped to $1 / 80$. Treatment which lasted for 9+ months was discontinued, and the patient is kept under regular follow-up where he is still asymptomatic.

Case 3, a 24 years old female, a known case of thalassemia trait, a school teacher. In November 2019, she was presented with a history of headache, joint pain and low-grade fever over a period of four weeks as mentioned in table 4 .

She was admitted to hospital and was treated with oral antibiotics. But her headache worsened and became more constant and diffuse and was associated with blurred vision and vomiting. On clinical examination, there was mild neck rigidity. Ophthalmology examination revealed bilateral optic disc swelling, and brain MRI showed features of increased ICP, while MRV was normal. A CSF analysis was performed, and the results were as follows: WBC $60 \mathrm{~mm}^{3}$ (lymphocytes $90 \%$ ), protein $115 \mathrm{mg} / \mathrm{dl}$, and glucose 30 $\mathrm{mg} / \mathrm{dl}$. ICP was $50 \mathrm{cmH}_{2} \mathrm{O}$ and PCR for tuberculosis in CSF was negative. The Brucella test (SAT) was positive with an antibody titer $1 / 320$. According to the above results, we commenced the patient on the same triple antibiotic regimen as in cases 1 and 2, plus acetazolamide. The latter was stopped after four weeks as papilledema resolved. 
In February 2020, a new CSF analysis was done with dramatic improvement: WBC $12 \mathrm{~mm}^{3}$ (lymphocytes $90 \%$ ), protein $68 \mathrm{mg} / \mathrm{dL}$, glucose $39 \mathrm{mg} / \mathrm{dL}$ and Brucella antibody titer dropped to $1 / 160$. Treatment was continued on the same protocol. CSF analysis was repeated in June 2020, coming back with complete resolution of CSF parameter: WBC $0-5 \mathrm{~mm}^{3}$, protein $23 \mathrm{mg} / \mathrm{dL}$, glucose $45 \mathrm{mg} / \mathrm{dL}$ and the Brucella antibody titer dropped to $1 / 80$. Treatment was discontinued, and on regular follow-up the patient remains asymptomatic.

Case 4, a 39 years old male, medically free, working as a soldier asmentioned in table 5. In June 2019, he was admitted to a peripheral hospital where he was diagnosed and treated as a case of partially treated bacterial meningitis. His laboratory data were recovered from that hospital with CSF analysis showing: WBC $150 \mathrm{~mm}^{3}$ (lymphocytes $65 \%$, neutrophils $35 \%$ ), protein $86 \mathrm{mg} / \mathrm{dL}$ and glucose 70 $\mathrm{mg} / \mathrm{dL}$. Brain MRI was reported as normal, and the patient was discharged after being treated for 14 days with intravenous antibiotics (ceftriaxone and vancomycin). Two weeks later after being discharged, the patient was readmitted to our neurology ward after representing with left-sided upper and lower limb weakness, fever and headache. His brain MRI showed acute ischemic infarction in the right posterior aspect of the right temporal lobe (Fig.1) . CSF analysis revealed high WBC count $85 \mathrm{~mm}^{3}$ (80\% lymphocytes, $20 \%$ neutrophils), protein $111 \mathrm{mg} / \mathrm{dL}$ and glucose 37 $\mathrm{mg} / \mathrm{dL}$. ESR was $35 \mathrm{~mm} / 1^{\text {st }} \mathrm{hr}$ and CRP was normal. The Brucella test (SAT) was positive with an antibody titer of 1/640. PCR for tuberculosis in CSF was negative.

The patient was commenced on intravenous Ceftriaxone $2 \mathrm{~g}$ IV daily for three weeks (replaced then by oral trimethoprim-sulfamethoxazole), rifampicin $600 \mathrm{mg} /$ daily orally and doxycycline $100 \mathrm{mg}$ bid/ daily for four months. Limb weakness has fully recovered with only mild residual weakness, and a follow-up CSF analysis with Brucella titer were done in December 2019 with the following results: WBC $25 \mathrm{~mm}^{3}$ (with all mononuclear cells), protein $58 \mathrm{mg} / \mathrm{dL}$, glucose $67 \mathrm{mg} / \mathrm{dL}$ and negative CSF culture, where the Brucella antibody titer was $1 / 160$. These results, in addition to the dramatic clinical improvement, indicated that treatment must be continued until CSF parameters returns to normal.

In July 2020, CSF analysis was repeated showing complete recovery, with nil WBC0-5 $\mathrm{mm}^{3}$, Protein 38 $\mathrm{mg} / \mathrm{dL}$ and Glucose $67 \mathrm{mg} / \mathrm{dL}$. CRP was normal, ESR $25 \mathrm{~mm} / 1^{\text {st }}$ hour, and Brucella antibody titer dropped to $1 / 20$. Treatment was discontinued after a total duration of 13+ months, with full clinical and laboratory recovery and the patient is on regular follow-up intervals since then.
Case 5, a 15 years old male, medically free, his family owned a big sheep farm as mentioned in table 6 . He was newly diagnosed with brucellosis with confirmed laboratory results at presentation, with a Brucella antibody titer 1/640 and isolated Brucella melitensis, for which he was given a course of antibiotics with doxycycline $100 \mathrm{mg}$ in combination with rifampicin $300 \mathrm{mg}$ each twice daily for 14 days. A few weeks later, he developed progressive bilateral lower and upper limb weakness (more severe in the lower limbs), associated with impaired sensation up to the level of the nipples, coupled with a history of progressive difficulty of urination. The latter was relieved via Foley's catheter insertion.

On examination, he was alert, oriented and cranial nerve examination was normal. Limb examination showed decreased power in both lower and upper limbs with $3 / 5$ and $4 / 5$, respectively. $\mathrm{He}$ was also found to have hyperreflexia with clonus and hypertonicity all over, but more evident in the lower limbs, in addition to diminished all modalities of sensation up to mid-thoracic level.

He was admitted to hospital in December 2018. Whole spine MRI showed hyperintensities at the spinal cord from C7-T1 with associated cord oedema Fig 2.

The CSF analysis of this patient showed $55 \mathrm{~mm}^{3} \mathrm{WBC}$ (95\% lymphocytes), high protein $200 \mathrm{mg} / \mathrm{dL}$, glucose $32 \mathrm{mg} / \mathrm{dL}$, with negative CSF culture and PCR for tuberculosis in CSF.

Patient was started on treatment with intravenous ceftriaxone 2 gram twice daily for three weeks, rifampicin $600 \mathrm{mg}$ twice daily and doxycycline $100 \mathrm{mg}$ twice daily, in addition to intravenous steroid (dexamethasone $4 \mathrm{mg}$ ) three times daily for 14 days which was then tapered off over one week.

Three weeks later, ceftriaxone was replaced with oral trimethoprim-sulfamethoxazole 400/80 mg twice daily, while the other medications were kept the same. Patient was having regular physiotherapy sessions at the rehabilitation center, and he started to improve over weeks.

In May 2019, Brucella antibody titer was $1 / 320$, and CSF analysis showed: WBC $28 \mathrm{~mm}^{3}$ (all mononuclear), protein $109 \mathrm{mg} / \mathrm{dL}$ and glucose $45 \mathrm{mg} / \mathrm{dL}$. Patient was kept on the same treatment.

In November 2019, Brucella antibody titer dropped to $1 / 160$, and CSF analysis showed: normal WBC, protein $76 \mathrm{mg} / \mathrm{dL}$ and glucose $65 \mathrm{mg} / \mathrm{dL}$. Limb weakness has improved and patient was walking independently, with mild residual hypertonicity in the lower limbs and urinary symptoms resolved entirely.

In June 2020, CSF analysis was done with normal WBC, Protein $45 \mathrm{mg} / \mathrm{dL}$ and Glucose $59 \mathrm{mg} / \mathrm{dL}$. CRP was normal, ESR $20 \mathrm{~mm} / 1^{\text {st }}$ hour, and his Brucella 
antibody titer was $1 / 20$. Whole spine MRI with contrast showed a complete resolution of spinal cord lesion. Treatment was stopped after $18+$ months and he is subjected to regular follow-up interval since then.

\section{Discussion}

Brucellosis is the most common zoonotic infection around the globe where it is still endemic in certain areas. In Jordan it is still highly endemic despite vaccination programs with extensive workshops and courses in cooperation with local and international organizations, to improve the knowledge and provide proper diagnostic tools and protocols. However, Brucella still has a significant impact on both health and economy.

Brucellosis is mostly acquired through consumption of unpasteurized milk and its products, or via direct contact with infected animals or animal products.

Neurobrucellosis can affect all parts of the nervous system [18], with variable mechanisms in direct or indirect pathways. In recent studies, inflammation is the hallmark of brucellosis despite the fact that the inflammatory process developing in brucellosis seems to be lower than with other types of bacteria [26, 33]. The chronic nature of the infection favors inflammation as the primary cause of tissue damage induced by indirect means, most probably through activation of the host's innate immune response [31], after recognition of Brucella antigens. Because of the ability of Brucella bacteria to rip-off the immune system and the wide varieties of different effects on the nervous system, neurobrucellosis can mimic a lot of other neurological diseases[32].

Neurobrucellosis still constitutes a diagnostic challenge; it is tough to suspect and recognize it at the earlier stages of the disease, which may lead to an unintentional delay in reaching an accurate diagnosis for up to 4-6 months, in range. Neurobrucellosis is a treatable disease, early diagnosis and treatment will result in a favorable outcome decreasing mortality which is reported to be between 0\%-27\%, and morbidity which depends on the neurological manifestations at presentation and the time of diagnosis [19].

In our neurobrucellosis case series, we attributed the approximate estimated time for establishing an accurate diagnosis to range between 2-4 months. We related this delay to the compilation of variable nonspecific symptoms and the lack of specific signs of neurobrucellosis. In our cases, there were neurological manifestations, developed either at an early or at a late stage after the disease has entered a chronic course. In case number four, there was a focal motor deficit resembling ischemic stroke, and in case number five there was transverse myelitis as a direct response to inflammation and vascular changes. In addition, the inflammatory results of their CSF analysis was enough to raise the possibility of neurobrucellosis, with the exclusion of other possible causes that have a similar effect in CSF like tuberculosis.

It is rare for neurobrucellosis to be complicated with papilledema[28, 29], which reflects an increase in ICP in response to the direct toxic effect of brucella, with high protein levels in CSF as a result of meningoencephalitis. In our cases which presented with optic disc swelling (case 1, 2 and 3), with lack of or mild meningeal signs and the result of CSF, we have extended the workup to look for the primary cause.

The workup in neurobrucellosis is broad, and the evaluation runs according to the patient's presentation and neurological findings. In those patients presented with optic disc swelling and focal neurological manifestations, brain images are mandatory, like computed tomography (CT) and magnetic resonance imaging (MRI \& MRV), done both at presentation and during follow-up. We performed Lumbar puncture in all cases initially, and at regular follow-up intervals every 3-4 months, to re-evaluate the results of CSF analysis and response to treatment. As Jordan is still considered a high endemic area for TB, another investigation was done to exclude Mycobacterium tuberculosis which can mimic neurobrucellosis. PCR in CSF was done for all patients with high protein combined with a low glucose level in CSF. The gold standard test for brucella was the standard agglutination test (SAT). The test was done on serum but not on CSF, where the antibody titer was followed up regularly side by side with the CSF analysis to monitor for treatment response and to decide when to stop the antibiotic regime. Isolation of Brucella species with growth in both CSF and serum cultures was positive in one case (case number 5), and negative in all of the remaining cases.

The diagnosis of neurobrucellosis is perplexing and challenging to all physicians [30], and in lack of specific diagnostic criteria, the clinical presentation, the results of investigations, in addition to the epidemiology information and the response to the antibiotics must be brought into considerations to reach the diagnosis of neurobrucellosis.

The suggested and applied criteria were efficient in the diagnosis of our neurobrucellosis cases, where almost all of the cases have fulfilled it. On the one hand, the challenge with this criteria was in the absence of specific signs and symptoms for neurobrucellosis, and on the other hand, the difficulty which faced us to isolate and grow the Brucella species either in CSF or serum. However, this proposed criteria was useful and an ideal to apply in such cases. 
In the treatment of our cases, we used the triple antibiotic regimen, doxycycline, rifampicin and a third-generation cephalosporin for three consecutive weeks, then it was substituted by trimethoprimsulfamethoxazole via oral route [20, 23, 24, 25,34,35]. In one patient, systemic steroid as an oral prednisolone was given for a short period because of cord oedema. We assessed the efficacy and response to this regime via clinical response and the dramatic resolution of symptoms, along with the biochemical reaction. In our cases, the success rate was high. The duration of treatment was determined according to the CSF analysis and serum brucella titer done on regular basis, where the treatment course was set initially to be in the range of 4 to 9 months [21, 22]. Although in cases number 2 and 3 the treatment duration was around nine months, still in the remaining cases it was extended to more than 12 months, until complete recovery of their CSF analysis and a low serum Brucella titer $<1 / 160$ was achieved.

\section{Conclusion}

Neurobrucellosis is still a staggering disease. The pathway to diagnose, treat and follow it up, is strenuous for both the physician and the patient. In our case series, all patients recovered entirely without relapse, and the applied criteria, investigations and a long duration of treatment with triple antibiotics all paid off. In our small group of neurobrucellosis cases, we recommend the use of the proposed criteria and the tripartite regime of antibiotics, as it showed excellent results of recovering and preventing mortality and morbidity.

\section{Acknowledgement}

We are gratefully thankful for all patients and their families, for their patience and cooperation. And we are grateful for all physicians in the neurology department in king Hussein medical center who participated in this work and their outstanding effort.

\section{References}

[1]. DrGeorgiosPappasaPhotiniPapadimitrioubNik olaosAkritidiscLeonidasChristouaEpameinond as VTsianosa https://doi.org/10.1016/S14733099(06)70382-6, the lancet, infectious disease, Volume 6, Issue 2, February 2006, Pages 91-99.

[2]. World Organization for Animal Health. Handistatus II: zoonoses (human cases): global cases of brucellosis in 2000. http://www.oie. int/hs2/gi_zoon_mald.asp?c_cont $=6 \&$ c_mald= $172 \&$ annee $=2000($ accessed Dec 13, 2005).

[3]. Epidemiological aspects of brucellosis in Jordan, Qasem M. Abu Shaqra, European
Journal of Epidemiology volume 16, pages 581-584(2000).

[4]. Issa H, Jamal M. Brucellosis in children in south Jordan. East Mediterr Health J 1999; 5: 895-902.

[5]. Human to human transmission of Brucella melitensis, Can J Infect Dis. 1995 May-Jun; 6(3): 153-155. DOI: 10.1155/1995/909404.

[6]. Akdeniz H, Irmak H, Anlar Ö, Demiröz AP. Central nervous system brucellosis: presentation, diagnosis and treatment. J Infect. 1998; 36:297-30, Journal of I qection (1998) $36,297-301$.

[7]. Neurobrucellosis, Neuroimaging Clinics of North America - November 2011 DOI: 10.1016/j.nic.2011.07.008 • Source: PubMed.

[8]. Neurobrucellosis: Clinical and Neuroimaging Correlation, American Journal of Neuroradiology • April 2004 Source: PubMed.

[9]. Neurobrucellosis: A neglected entity? An update from tertiary care Neurocentre of South East Asia, journal of neurological sciences, https://doi.org/10.1016/j.jns.2020.116683.

[10]. Neurobrucellosis: Clinical and Diagnostic Features, Clinical Infectious Diseases, Volume 56, Issue 10, 15 May 2013, Pages 1407-1412, https://doi.org/10.1093/cid/cit072.

[11]. McLean DR, Russell N, Khan MY. Neurobrucellosis: clinical and therapeutic features, Clin Infect Dis, 1992, vol. 15 (pg. 582-90).

[12]. Shakir RA, Al-Din AS, Araj GF, Lulu AR, Mousa AR, Saadah MA. Clinical categories of neurobrucellosis. A report on 19 cases, Brain, 1987, vol. 110 Pt 1(pg. 213-23).

[13]. Ay S, Tur BS, Kutlay S. Cerebral infarct due to meningovascular neurobrucellosis: a case report, Int J Infect Dis, 2010, vol. 14 suppl 3(pg. e202-4).

[14]. Sanchez-Sousa A, Torres C, Campello MG, et al. Serological diagnosis of neurobrucellosis, J Clin Pathol, 1990, vol. 43 (pg. 79-81).

[15]. Akdeniz H, Irmak H, Anlar O, Demiroz AP. Central nervous system brucellosis: presentation, diagnosis and treatment, J Infect, 1998, vol. 36 (pg. 297-301).

[16]. Vasculitis and neurobrucellosis: Evaluation of nine cases using radiologic findings 2018, Brain and Behavior. 2018;8:e00947.

DOI: $10.1002 / b r b 3.947$.

[17]. Young EJ. Brucella species. In: Douglas M, editor. Bennett's principles and practice of 
infectious diseases. 6th ed. Churchill Livingstone Co; 2005. p. 2669-73.

[18]. Bodur H, Erbay A, Akinci E, Colpan A, Cevik MA, Balaban N. Neurobrucellosis in an endemic area of brucellosis, Scand J Infect Dis, 2003, vol. 35 (pg. 94-7).

[19]. Pappas G, Akritidis N, Christou L. Treatment of neurobrucellosis: what is known and what remains to be answered, Expert Rev Anti Infect Ther, 2007, vol. 5 (pg. 983-90).

[20]. Erdem H, Ulu-Kilic A, Kilic S, et al. Efficacy and tolerability of antibiotic combinations in neurobrucellosis: results of the Istanbul study, Antimicrob Agents Chemother, 2012, vol. 56 (pg. 1523-8).

[21]. Short course treatment of neurobrucellosis, a study in Iran, International Journal of Infectious Diseases 16S (2012) e158-e316, doi.org/10.1016/j.ijid.2012.05.793.

[22]. Treatment of neurobrucellosis with a combination of ceftriaxone, rifampicin and doxycycline (a study on ten cases), doi.org/10.1016/S0399-077X(96)80124-7.

[23]. Neurobrucellosis: clinical, diagnostic, therapeutic features and outcome. Unusual clinical presentations in an endemic region, doi.org/10.1016/S1413-8670(11)70140-4.

[24]. Comparative trial of co-trimoxazole versus tetracycline-streptomycin in treating human brucellosis, J Infect Dis, 1985 Dec;152(6):1358-9. doi: 10.1093/infdis/152.6.1358.

[25]. Brucella abortus-activated microglia induce neuronal death through primary phagocytosis, PMID: 28398652 DOI: 10.1002/glia.23149.

[26]. Myelitis and Polyradiculoneuropathy With Severe Pain Unusual Neurological Manifestations as Presenting Symptoms of Brucellosis, The Neurologist Volume 23, Number 4, July 2018, doi.org/10.1097/nrl.0000000000000184

[27]. Neurobrucellosis Presenting as Pseudotumor Cerebri: First Report from Oman, DOI 10.5001/omj.2017.96.

[28]. A Case of Neurobrucellosis That Mimicks Increased Intracranial Hypertension Syndrome Article in Turk Noroloji Dergisi - December 2014, DOI: 10.4274/tnd.78736.

[29]. Neurobrucellosis, Marine Le Dudal Fabrice Chrétien Grégory Jouvion. https://doi.org/10.1002/9781119467748.ch39.

[30]. In Vitro Brucella suis Infection Prevents the Programmed Cell Death of Human Monocytic
Cells, Antoine Gross, Annie Terraza, Safia Ouahrani-Bettache, Jean-Pierre Liautard, and Jacques Dornand.

dx.doi.org/10.1128\%2Fiai.68.1.342-351.2000.

[31]. Glial Cell-Elicited Activation of Brain Microvasculature in Response to Brucella abortus Infection Requires ASC Inflammasome-Dependent IL-1 $\beta$ Production, doi.org/10.4049/jimmunol.1500908.

[32]. Immunopathology of Brucella infection, doi.org/10.2174/1574891x11308010005.

[33]. Treatment of human brucellosis: systematic review and meta-analysis of randomised controlled trials, BMJ 2008; 336 doi: https://doi.org/10.1136/bmj.39497.500903.25 (Published 27 March 2008).

[34]. Systematic review and meta-analysis of randomized clinical trials in the treatment of human brucellosis, Julián Solís García del Pozo 1, Javier Solera, PMID: 22393379 PMCID: PMC3290537

DOI: 10.1371/journal.pone.0032090.

[35]. Neurobrucellosis: Clinical and Diagnostic Features Article in Clinical Infectious Diseases $\bullet$ February 2013.

DOI: 10.1093/cid/cit072. Source: PubMed. 